TIP Periodica Polytechnica

Electrical Engineering and Computer Science

58(2), pp. 43-53 (2014)

DOI: 10.3311/PPee.7477

Creative Commons Attribution (i)

RESEARCH ARTICLE

\section{Nonlinear electro-thermal and light output modeling and simulation of OLEDs}

Ernő Kollár / László Pohl / András Poppe / Zsolt Kohári

RECEIVED 18 MARCH 2011; ACCEPTED 26 JUNE 2012

\section{Abstract}

Lighting application of Organic Light Emitting Diodes (OLEDs) has been forecasted and such applications appear already in the high end segment of the market. These devices should provide homogeneous luminance - homogeneity is one of the quality metrics of such devices. Local light generation depends on both the local temperature and the local voltage drop across the light emitting polymer(s) in the device. Due to the large area of these devices the coupled optical, electrical and thermal simulation problem is of distributed nature. Electrical characteristics of organic semiconductor materials used in OLED devices are nonlinear, and their nonlinear temperaturedependence is significant. Measurement and temperature-dependent electrical and optical modeling of OLEDs is presented in this study. The paper addresses the special needs of OLEDs in distributed electro-thermal field simulation. These needs are not handled appropriately yet in the widely used, commercially available simulation tools. However the latest version of our SUNRED field solver algorithm is capable of handling these coupled, non-linear optical-electrical-thermal problems. The new features of the algorithm are demonstrated by modeling some research OLED samples available to us in the Fast2Light project - this way simulation results are compared against measured data. The results suggest that the models and the simulation tool can be used well in OLED design.

\section{Keywords}

OLED · modeling · field simulation · electro-thermal . luminance

\section{Ernő Kollárkollar@eet.bme.hu \\ László Pohl pohl@eet.bme.hu \\ András Poppe poppe@eet.bme.hu \\ Zsolt Kohári kohari@eet.bme.hu}

Department of Electron Devices, Faculty of Electrical Engineering and Informatics, Budapest University of Technology and Economics, Magyar tudósok krt. 2., H-1117 Budapest, Hungary

\section{Introduction}

Solid-state light emitting device technology has made tremendous progress in the last decade. After the development of the non-organic blue and white Light Emitting Diodes (LEDs) [1, 2] the road led straight to the solid-state lighting applications. Reports about developments [3] describe 62,000 hours lifetime for blue OLEDs of an operating brightness of $400 \mathrm{~cd} / \mathrm{m}^{2}$ thus, the development of lighting purpose OLED devices is expected to step to market phase from experimental phase [4].

Research until now has mainly been motivated by glass-based organic LED displays [5], where the aspects of high contrast, wide viewing angle and good response time are critical. In our research project called Fast2Light [6] the purpose is to develop a novel, cost-effective, high-throughput, roll-to-roll, large area deposition process for fabricating light-emitting polymerOLED foils for intelligent lighting applications.

As of today, luminous flux and efficacy of organic devices is small compared to conventional light sources, so their surface must be much greater to provide comparable light output: in the Fast2Light project the targeted surface is $60 \times 60 \mathrm{~cm}^{2}$. This area must produce homogeneous light (the uniformity of the luminance on the surface should be over 95\%), which requires homogeneous current densities in the device. Despite the large surface, the operating power density of the device is significant. The polymer substrate and the materials used in the OLEDs have bad electrical and heat transfer properties that leads to another problem: the dissipation. Organic materials are very sensitive to overheating thus, their temperature must be maintained low, therefore proper cooling solutions should be provided in their application environment.

The above mentioned problems make essential the usage of coupled electrical-thermal field simulation. Available OLED simulation methods and tools deal with electrical and optical simulation of the devices e.g. [7-10] but thermal investigation means a problem. Commercial FEM simulation tools such as Ansys [11] can handle electrical and thermal fields, they can determine potential and temperature fields accurately but heatflow and current density calculation is a problem for them 
especially when these fields are coupled through special nonlinear characteristics. In OLEDs the key factor for providing homogeneous light emission is the uniformity of the potential drop across the light emitting polymer layer. In conventional device constructions transparent, yet electrically conductive anode electrode is needed.

Traditionally Indium Tin Oxide (ITO) is used for this but its electrical conductivity is not sufficient to provide the required potential uniformity over a large area. The workaround for this problem is the application of a metal grid as a shunting network in the transparent anode. Narrow and widely spaced wires allow the devices emit more light but in narrow wires the electric current density can be so high that it can cause degradation, which means that calculation of electrical current densities is also an important requirement. Park et al. presented a simple and well usable method for metal grid calculations based on lumped element SPICE simulation [12] but this method lacks the handling of thermal effects. The overall electrical behavior of an OLED device is determined by the coupled, distributed electro-thermal properties of the device where the I-V characteristics and the temperature dependence of the LEP (light emitting polymer) layer(s) are considered in a consistent way. The lack of such simulation code motivated us to extend our existing FDM based thermal simulation tool and algorithm, SUNRED (SUccessive Node REDuction) [13].

In operation the ambient temperature can change in a wide range, and the placing, mounting and cooling of the OLED significantly affect the heat flow of the dissipated power thus, the temperature distribution in the device. The efficiency of light emission and the electrical characteristics of organic materials are strongly and nonlinearly temperature-dependent, so linear OLED device models are not appropriate for the design of packaging and for examinations of the operation in real environment.

In this paper the OLEDs and their simulation needs are surveyed first, then an OLED model derived from our measurement results is described and an overview of the new, extended SUNRED algorithm is provided. The measurement of the OLED samples available to us is also discussed and the method based on [14] for creating nonlinear electro-thermal and optical device model equations for OLEDs is presented. Section 5 presents the implementation of nonlinear device models in the SUNRED field solver, including the split between the emitted optical power and the dissipated power. Finally the measured characteristics of the sample OLEDs with the results gained by the solution of the model equations and with the simulation results are compared. The simulated radiance and luminance distribution of the device is also demonstrated.

\section{Problem statement and background}

\subsection{Organic light emitting devices}

Although conventional LEDs based on III-V semiconductors (AlInGaP, InGaN) achieve bright emission with sufficient quantum efficiency for the visible spectral region, their use for large area general lighting applications is unlikely due to the fabrication cost and packaging issues. Organic semiconductors however, show good charge carrier transport properties as well as are excellent candidates for cheap and highly effective alternatives for large area applications [15].

Organic Light Emitting Diodes (see Fig. 1) are thin-film multi-layer devices consisting of a substrate foil, film or plate (rigid or flexible), an electrode layer, layers of active materials, a counter electrode layer, and a protective barrier layer. At least one of the electrodes must be transparent to light [15]. The prevailing transparent electrode material is ITO that is a bad electrical and thermal conductor. The active area is a semiconductor junction of at least two layers: PEDOT (Poly(3,4-ethylenedioxythiophene)) and organic light emitting layer. Multilayer OLEDs can have more than two layers to improve device efficiency and provide appropriate light emission spectrum. The light emitting layer can be built of small organic molecules, light emitting polymer (LEP) or phosphorescent materials.

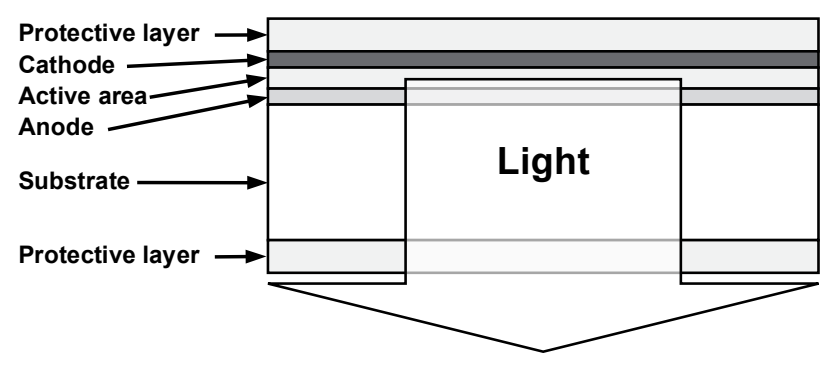

Fig. 1. Simplified layer structure of OLED

\subsection{Simulation needs}

The poor electrical conductance of the anode layer and the large area of the targeted lighting device $\left(60 \times 60 \mathrm{~cm}^{2}\right)$ raise the need for electrical simulation, to predict the voltage drop over the large surface. Lumped electrical circuit models are not appropriate for this purpose, distributed simulation must be applied. In its physical nature, the electrical simulation problem resembles thermal simulation. The electrical potential distribution can be studied using a thermal simulation tool utilizing the electrical-thermal analogy [16]. $1 \mathrm{~W}$ dissipation corresponds to $1 \mathrm{~A}$ electrical current. In this case, $1 \mathrm{~K} / \mathrm{W}$ thermal resistance represents $1 \mathrm{~W}$ of electrical resistance and the simulated temperature corresponds to the potential distribution, consequently, $1 \mathrm{~K}$ temperature difference corresponds to $1 \mathrm{~V}$ of potential drop.

Thermal simulations aim at examining the temperature distribution over the surface and inside the layer structure to ensure the correct functioning of the device by avoiding e.g. hot spot formation thus, avoiding local overheating, which may result in dark dots. The predicted surface temperature distribution can be verified by IR thermal measurement. 
A validation technique of the OLED simulation models includes the following steps:

- measurement of I-V characteristics of OLEDs in a temperature controlled environment; identification of the temperature sensitivity of the forward voltage as a temperature sensitive parameter (TSP);

- measurement of the temperature sensitivity of the light output

- measurement of the energy conversion efficiency (providing input for correction IR measurement results)

- IR measurements to validate the thermal simulation models.

The main objectives of the simulation are the following:

- to predict the voltage drop in the large area OLEDs to allow design of appropriate shunting nets,

- to calculate joule heating in the OLEDs,

- to end up with a temperature distribution of the large area OLEDs, based on the calculated dissipation map.

- to determine electrical behavior, potential and current density distribution, uniformity of the luminance at various ambient temperatures and thermal boundary conditions.

Thermal boundary conditions are governed by the placing and mounting of the OLED device. If the OLED foil is mounted directly on the wall or on the plastic false ceiling, the resulting temperature gradient can significantly affect the potential distribution and thus, the currents in the device. The amount of emitted light is proportional to the current density. The efficiency of the device is also influenced by the temperature. If the device is mounted on metal or other good heat conductor surface, the temperature gradient and thus, the luminous flux gradient will be smaller. A proper electro-thermal simulation can provide the temperature and current map of the large-area OLED that is essential for the design of the appropriate cooling and mounting.

\subsection{OLED model}

In our earlier publication [14] we have demonstrated an OLED model described by a power function in the forward voltage region (1) where the current of the device is expressed by the biasing voltage. This model seems to fit better for OLEDs than the Shockley diode equation (2).

$$
\begin{gathered}
I_{\text {OLED }}=b_{\text {LOW }} \cdot U^{m_{\text {LOW }}}+b_{H I G H} \cdot U^{m_{\text {HIGH }}} \\
I_{\text {diode }}=I_{0}\left(e^{\frac{U}{n V_{T}}}-1\right)
\end{gathered}
$$

Parameter $b, m$ and $I_{0}$ are determined by the structure, material and temperature of the device. $U$ is the biasing voltage; $V_{T}$ is the thermal voltage $(k T / q) ; n$ is the ideality factor. The $L O W$ and $H I G H$ subscripts indicate the forward bias voltage range where the power function is effective (see Fig. 5).
In real applications the OLED is either turned on and gives light, operating in the $H I G H$ range, or it is turned off. The bias domain where the $L O W$ part is significant can be ignored because the device does not emit perceivable amount of light, so instead of eq. (1) the following simplified equation can be used:

$$
I_{O L E D}=b \cdot U^{m}
$$

The parameters $b$ and $m$ are temperature, structure and material dependent.

\subsection{SUNRED model}

SUccessive Node REDuction is a solution method for the Finite Differences Method (FDM) field simulation models [17]. The applicable fields are defined by partial differential equations (PDE-s): Laplace-equation and Poisson's equation [18]. Different implementations of the SUNRED algorithm can solve thermal, electrostatic or electro-thermal fields $[13,19]$. OLED design requires a special version of the electro-thermal simulator where nonlinear; temperature dependent electrical characteristics can also be used in the simulation.

Electro-thermal fields in the steady-state (DC) case can be described by four partial differential equations [16]. Finite differences methods approximate the solution of PDE-s by replacing the differential equations with difference equations. In one dimension this means

$$
f^{\prime}(a) \approx \frac{f(a+x)-f(a)}{x}
$$

By decreasing $x$ the approximation of the differentials becomes more accurate. The FDM method divides the examined geometrical domain into a finite number of simulation cells and calculates the (4) difference in the center of each section. In 2 or $3 \mathrm{D}$ this division results in a finite differences grid as shown in Fig. 2(a). Finite differences can be modeled as resistors between two neighboring nodes. The SUNRED method divides each resistor into two parts as illustrated by Fig. 2(b). $E^{\prime}, W^{\prime}, \ldots$ etc. denote East, West etc. neighboring nodes, $C$ indicates the center node. According to the thermal-electrical analogy, in thermal case the resistors are thermal resistances.

The thermal and the electrical fields are mapped to coupled networks; the coupling (dissipation) is realized as controlled current sources (Fig. 2(c)). The Successive Node Reduction algorithm solves this network for the given boundary conditions, electrical excitations and thermal loads, and determines all the voltages/temperatures and current densities/heat fluxes [19]. The base algorithm is a direct solution method by its nature; there is no need for iteration but since both the electro-thermal coupling and the OLED equations are nonlinear, iteration is inevitable. This internal iteration however, requires much less steps than an inherently iterative solution method. 


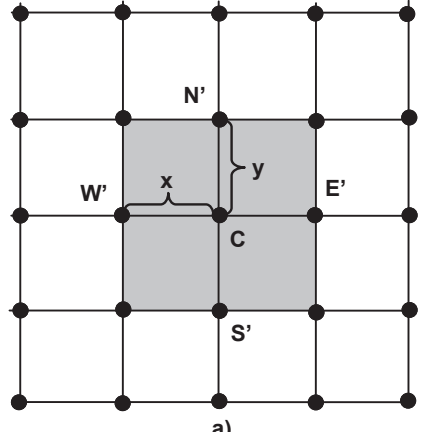

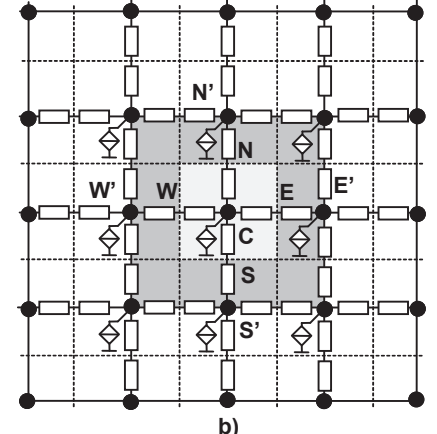

b)

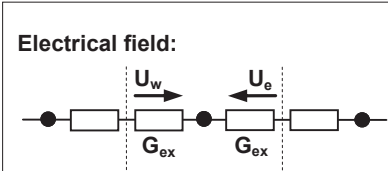

Thermal field:

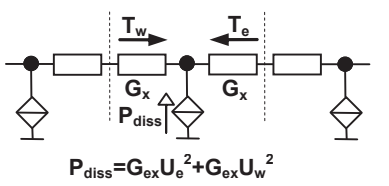

c)

Fig. 2. (a) Finite differences grid in 2D (b) SUNRED model in 2D (c) SUNRED electro-thermal coupling in 1D.

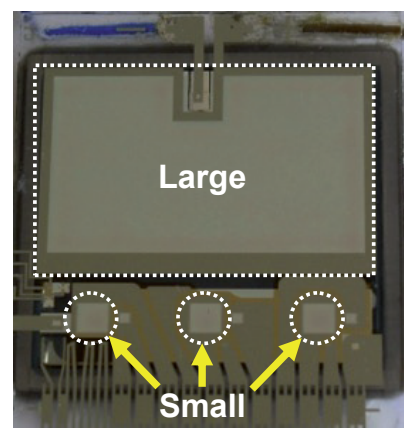

a)

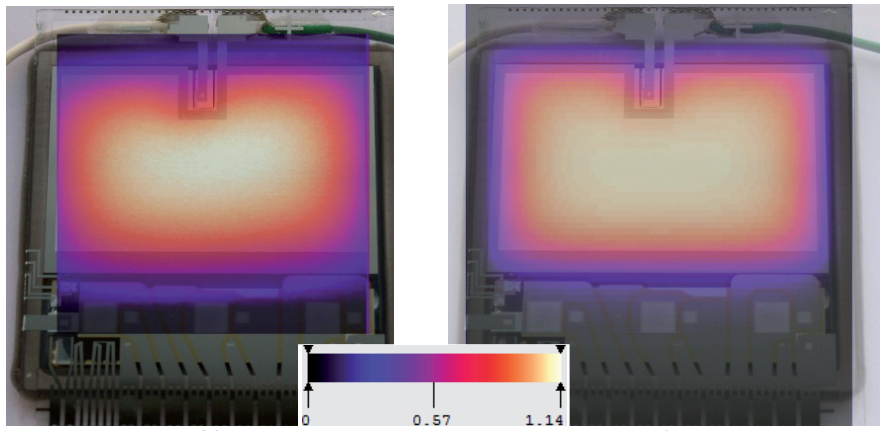

c)

Fig. 3. (a) A sample device with the investigated OLEDs. (b) Measured and (c) simulated surface temperature distribution at a heating power level of 565 $\mathrm{mW}$, with temperature scale.

\section{Measurement arrangement}

Proprietary OLED devices (Fig. 3(a)) realized on glass substrate were used for the measurements, modeling and simulations. Individual OLED pixels and a larger light emitting area were available on the sample device. Models presented in this paper refer to the small-sized OLEDs marked by arrows in Fig. 3(a). The size of these devices was approximately $3 \times 3 \mathrm{~mm}^{2}$ (active area was $9 \mathrm{~mm}^{2}$ ). Models were validated by the simulation of the "Large" OLED. The size of this device was approximately $33 \times 21 \mathrm{~mm}^{2}$ (active area was $564 \mathrm{~mm}^{2}$ ).

The surface of the OLED device was coated with a lightabsorbing paint ("black paint") for the IR measurements. Since this paint completely absorbs the light emitted by the OLED, it results in additional heating of the device surface. Thus, the IR measurements resulted in a map of somewhat elevated temperature values. To calculate the temperature map of the surface under normal operating conditions (without coating), the overall energy efficiency had to be measured. We used the TERALED measuring equipment for this purpose [20]. The measured and the simulated temperature map can be seen in Fig. 3(b) and (c).

I-V characteristics of OLEDs can be measured with conventional laboratory equipment. At our laboratory such measurements can be carried out in temperature controlled environment, with GPIB and RS232 controlled equipment. The measured device was attached to a thermostated cold-plate. Our measurement setup is outlined in Fig. 4.

As a result of the strong cooling, the temperature rise of the LEP of the small OLED remained under one degree at maximum dissipation, and that of the large OLED was slightly above one degree. That means the temperature rise does not cause significant inaccuracy in the model and in the validation.

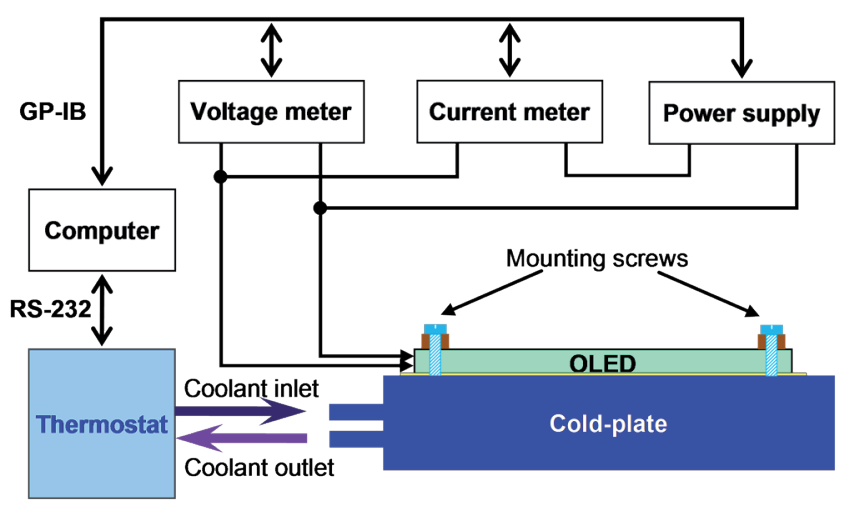

Fig. 4. Measurement setup for measuring I-V characteristics of OLEDs 
Radiometric and photometric measurements were performed, complying with the recommendations of the total flux measurement method for LEDs according to the CIE 127-2007 document [21]. For these measurements the TERALED equipment was used (Fig. 5). The equipment measures the total radiant flux or the total luminous flux of the DUT OLED device as a function of current. The measured quantity depends on the type of the detector. The temperature of the device is set by the temperature of the cold plate.

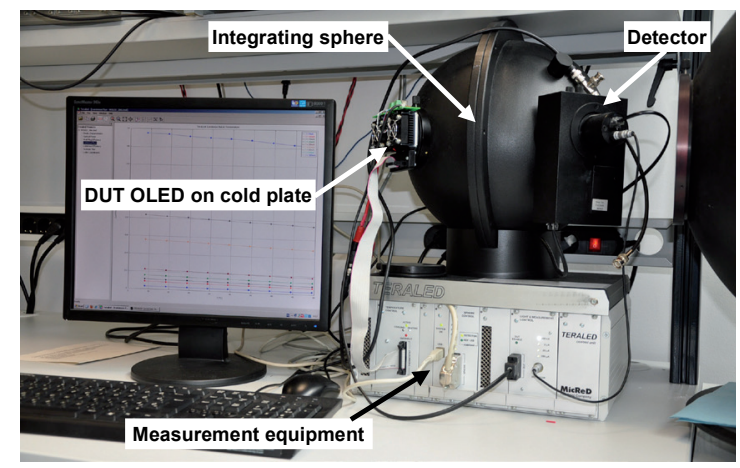

Fig. 5. Measurement setup for measuring I-L characteristics of OLEDs

\section{Model of the sample OLEDs \\ 4.1 Electro-thermal model}

The determination of the $b$ and $m$ parameters of (3) will be presented through the example of the measured sample devices. The parameters are temperature, structure and material dependent. The investigated samples do not differ in materials and structure except the thickness of the LEP layer: $60 \mathrm{~nm}$ and $80 \mathrm{~nm}$ respectively. Thus, only the temperature dependency should be used as parameter of $b$ and $m$ in the model:

$$
I_{O L E D}(T)=b(T) \cdot U^{m(T)}
$$

The I-V characteristics were measured between $5^{\circ} \mathrm{C}$ and $50^{\circ} \mathrm{C}$ in $5^{\circ} \mathrm{C}$ steps, and biasing voltage from $2 \mathrm{~V}$ in $0.01 \mathrm{~V}$ steps to the allowed maximum voltage at the specific temperature, which was determined by the allowed maximum current density of $22 \mathrm{~mA} / \mathrm{cm}^{2}$ specified by the manufacturer. We have measured all three small sized OLEDs on each sample. Fig. 6 presents curves measured at $25^{\circ} \mathrm{C}$ for samples with a LEP thickness of $60 \mathrm{~nm}$ and $80 \mathrm{~nm}$. In our case study simulation models were generated for the voltage range between $4 \mathrm{~V}$ and the limit voltage, which varied in the range of $5 . .6 \mathrm{~V}$, depending on the temperature. Fig. 7 shows the measured I-V curves of an OLED sample with $60 \mathrm{~nm}$ LEP. The current increases with increasing temperature, and the limit voltage decreases from $5.87 \mathrm{~V}$ at $10^{\circ} \mathrm{C}$ to $4.9 \mathrm{~V}$ at $50^{\circ} \mathrm{C}$.

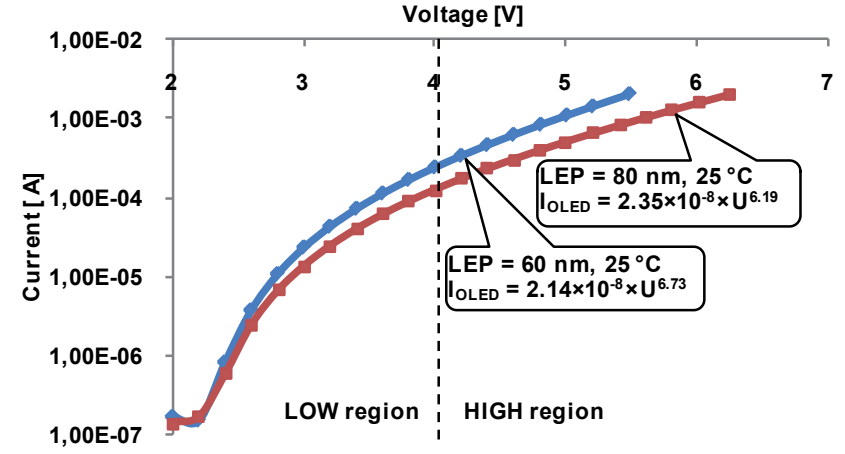

Fig. 6. The effect of the LEP thickness; forward I-V characteristics at $25^{\circ} \mathrm{C}$ and the fitted power functions

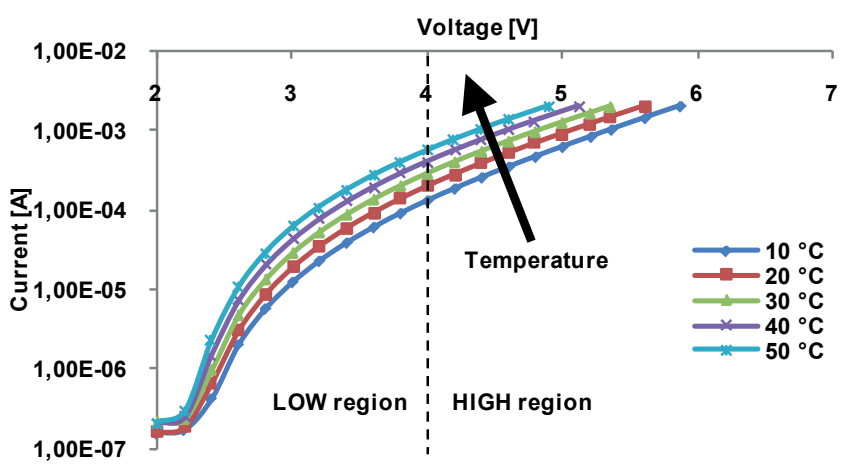

Fig. 7. The effect of the temperature change; forward I-V characteristics of a $60 \mathrm{~nm}$ LEP thickness OLED sample. (For clarity of the diagrams, only every 20 voltage value is plotted.)

To obtain the $b$ and $m$ parameters as function of the temperature, power functions were fitted to the I-V curves at each measured temperature (Fig. 6 comments). A generic quadratic expression according to equations (6a) and (6b) was fitted to each curve - resulting in a good approximation: variance was better than 0.998 in all cases. The temperature dependence of the identified $b$ and $m$ parameters is shown in Fig. 8 and 9 respectively.

$$
\begin{gathered}
b=b_{2} T^{2}+b_{1} T+b_{0} \\
m=m_{2} T^{2}+m_{1} T+m_{0}
\end{gathered}
$$

Using these, the resulting model equations for the sample OLEDs are:

$$
\begin{aligned}
& I_{\text {OLED, } 60 \mathrm{~nm}}(U, T)= \\
& \quad\left(0.397 \cdot T^{2}-5.47 \cdot T+94.2\right) \cdot 10^{-10} \cdot U^{1.410^{-4} \cdot T^{2}-2.55 \cdot 10^{-2} \cdot T+7.28} \\
& I_{\text {OLED }, 80 \mathrm{~nm}}(U, T)= \\
& \quad\left(0.42 \cdot T^{2}-5.75 \cdot T+109\right) \cdot 10^{-10} \cdot U^{1.16 \cdot 10^{-4} \cdot T^{2}-2.23 \cdot 10^{-2} \cdot T+6.68}
\end{aligned}
$$




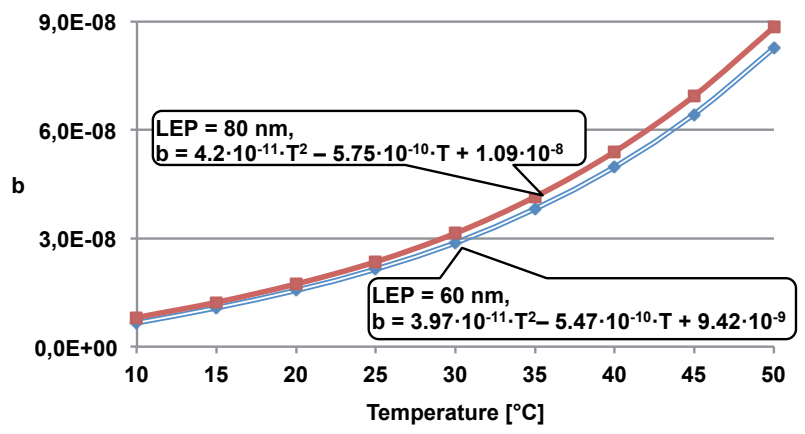

Fig. 8. The effect of the LEP thickness and temperature on the $b$ factor

\subsection{Optical model}

Current to radiant flux and current to luminous flux curves of the large OLEDs were measured from $10 \mathrm{~mA}$ to $120 \mathrm{~mA}$ in the $9^{\circ} \mathrm{C}$ to $49.6^{\circ} \mathrm{C}$ range in about $5^{\circ} \mathrm{C}$ steps. Some of the measured curves can be seen in Fig. 10 for the $60 \mathrm{~nm}$ and $80 \mathrm{~nm}$ LEP thickness samples. Light emission curves on the small OLEDs were not measured because the level of flux they emitted was below the sensitivity limit of our equipment.

For both the $60 \mathrm{~nm}$ and $80 \mathrm{~nm}$ LEP thickness samples the curves have two linear parts and there is a break between 29 $\mathrm{mA}$ and $41 \mathrm{~mA}$. Our simple model ignores the low current part because in practice high light output is expected from lighting purpose OLEDs.

Temperature-dependence of the radiant flux in the $41 \mathrm{~mA}$ to $120 \mathrm{~mA}$ range is $9 \%$ to $4 \%$ for the $60 \mathrm{~nm}$ LEP thickness samples and $9 \%$ to $3 \%$ for the $80 \mathrm{~nm}$ LEP OLEDs; decreases while the current increases. For lower currents the change reaches 16$17 \%$. Luminous flux changes from $12 \%$ to $6 \%$ and $11 \%$ to $5 \%$ in the higher current range and reaches $21-22 \%$ at lower currents. The change in luminous flux is higher than in radiant flux because the color spectrum of the OLED light is also changing. The change in the luminous flux is the more important because it fits to the sensitivity of human eye.

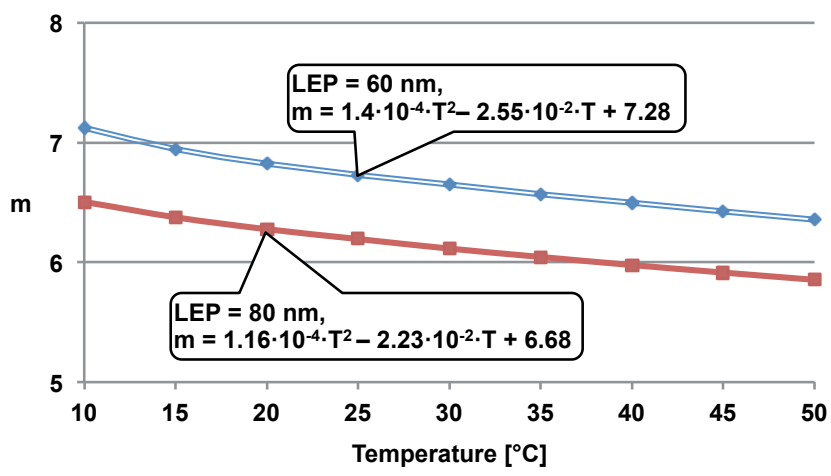

Fig. 9. The effect of the LEP thickness and temperature for the $m$ exponent

Model generation for the temperature-dependence of OLEDs is of two steps:

(I) Fitting a linear equation for each I-L characteristic. Equation (8a) and (8b) describes the fitted curves where $\phi_{V}$ is the radiant flux, $\phi_{e}$ is the luminous flux, $I$ is the current, $T$ is the temperature, $m r, b r, m l, b l$ are parameters.

$$
\begin{aligned}
& \varphi_{V}(I, T)=m r(T) \cdot I+b r(T) \\
& \varphi_{e}(I, T)=m l(T) \cdot I+b l(T)
\end{aligned}
$$

(II) Finding the appropriate $m r(T), b r(T), m l(T)$ and $b l(T)$ functions.

Fig. 11 presents the temperature-dependence of eq. (8) parameters. Linear approximation was chosen for each parameter and the fitted linear curves with their equations are also drawn in the figures. The resulting model equations for the sample large OLEDs are the following:

$$
\begin{aligned}
& \varphi_{V 60 n m}(I, T)= \\
& \quad\left(0.0393-T \cdot 2.51 \cdot 10^{-5}\right) \cdot I-\left(1.70 \cdot 10^{-4}+T \cdot 2.16 \cdot 10^{-6}\right)
\end{aligned}
$$

$$
\varphi_{e 60 n m}(I, T)=
$$

$$
(12.3-T \cdot 0.011) \cdot I-\left(0.0547+T \cdot 8.67 \cdot 10^{-4}\right)
$$
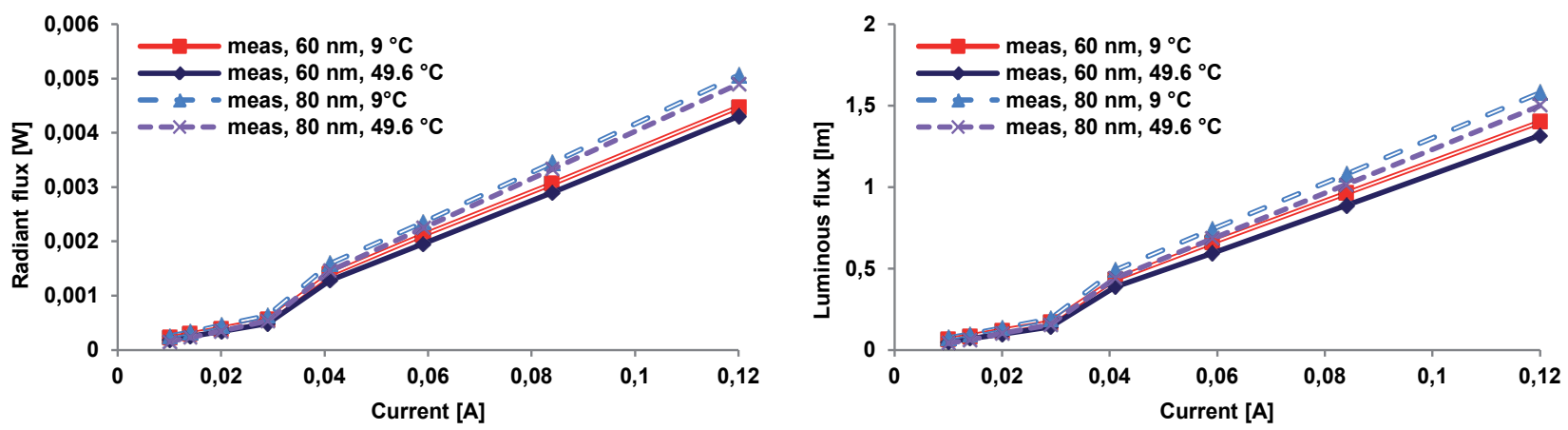

Fig. 10. I-L characteristics: the effect of the temperature change on light emission of the large OLEDs 


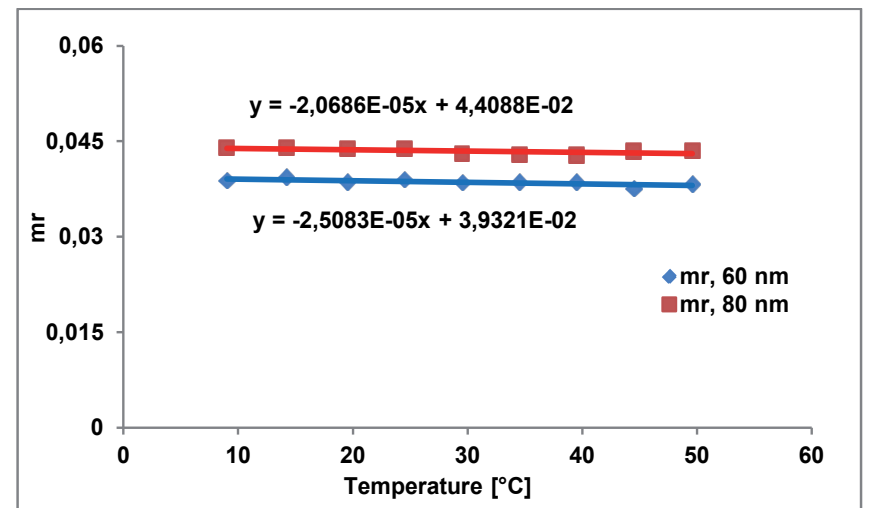

a)

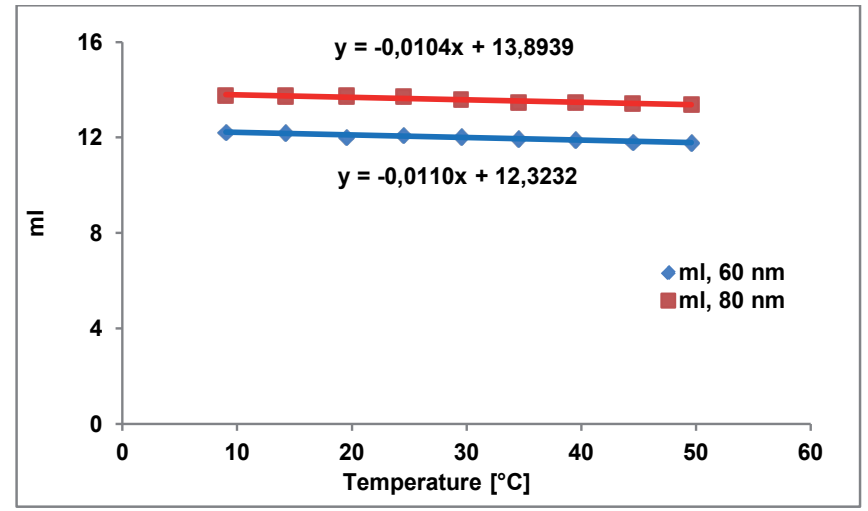

c)

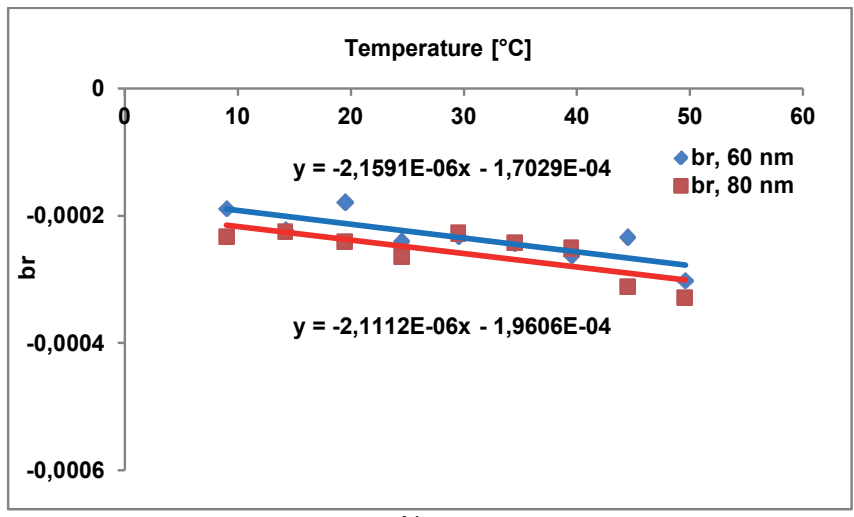

b)

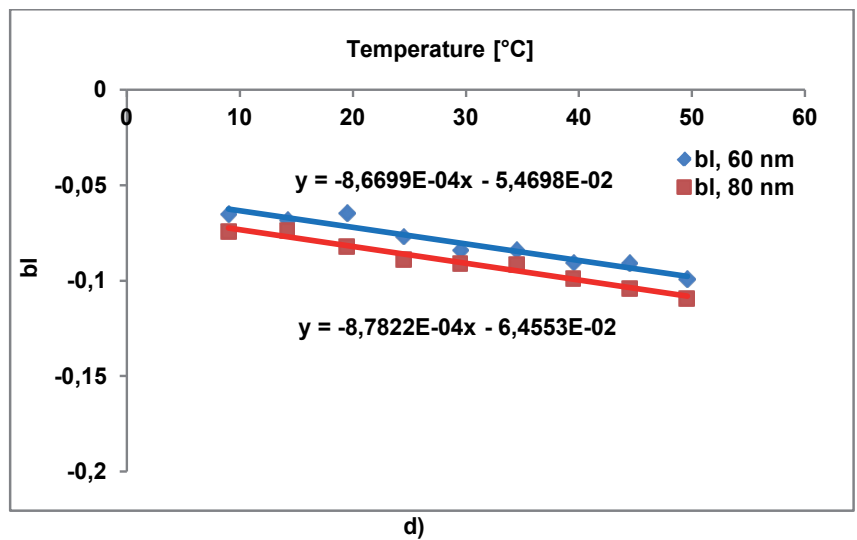

Fig. 11. Temperature-dependence of parameters of eq. (8) in case of the large OLEDs. Measured points and fitted linear curves.

$\varphi_{V 80 \mathrm{~nm}}(I, T)=$

$\left(0.0441-T \cdot 2.07 \cdot 10^{-5}\right) \cdot I-\left(1.96 \cdot 10^{-4}+T \cdot 2.11 \cdot 10^{-6}\right)$

$\varphi_{e 80 n m}(I, T)=$

$(13.89-T \cdot 0.010) \cdot I-\left(0.0646+T \cdot 8.78 \cdot 10^{-4}\right)$

\section{Semiconductor junction in SUNRED}

Semiconductor junction is a thin, electrically strongly nonlinear interface between two material layers. We modeled the junction with a nonlinear resistor [22]. Our solution can be seen in Fig. 12. Semiconductor junction is modeled with a series resistor between the two adjacent material regions ( $n$ and $p$ type).

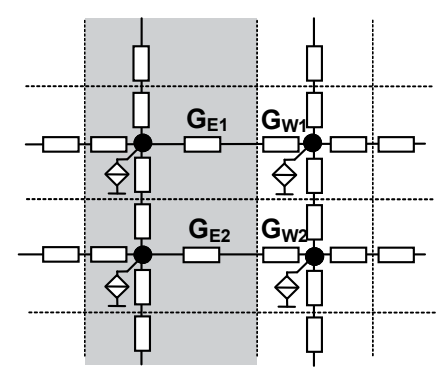

a)

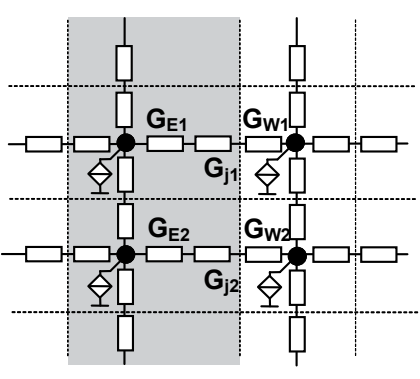

b)
Fig. 12. SUNRED model (a) normal materials (b) Semiconductor junction
Value of the resistor is derived from equation (3) by expressing the conductance. Equation (10a) shows the conductance of the full semiconductor junction while eq. (10b) presents the junction conductance of one cellside where $A_{\text {junction }}$ is the area of the full semiconductor junction of the device and $A_{\text {cell }}$ is the area of one cell side.

$$
\begin{gathered}
G_{j_{-} O L E D}=\frac{I_{\text {OLED }}}{U}=\frac{I_{\text {OLED }}}{\sqrt[m]{I_{\text {OLED }} / b}} \\
G_{j_{-} \text {cell }}(I)=\frac{I_{\text {cell }}}{\sqrt[m]{\frac{A_{\text {junction }}}{A_{\text {cell }}} I_{\text {cell }} / b}}
\end{gathered}
$$

Parameters $b$ and $m$ are implemented in SUNRED algorithm with the temperature dependent quadratic formulae defined by (6a) and (6b), respectively - the coefficients for (6a) and (6b) are input parameters for the simulation.

Solution of a nonlinear network requires iterative computation. We have chosen the successive approximation algorithm [23] because it was the simplest to implement. Other algorithms can be faster or their convergence might be better but their usage would have required major changes in our existing code. For example the popular Newton-Raphson method [23] necessitates the calculation of the Jacobian matrices. 
The purpose of OLED is to emit light so a part of the input power does not heat up the device. In the SUNRED model we handle the local energy conversion efficiency. Such, that the locally emitted optical power is subtracted from the calculated Joule heating.

\section{Results and validation}

Solution of (7) model equations provide I-V characteristics at any required temperature for the investigated device. These characteristics will change if the ambient (i.e. thermal boundary conditions) or the structure of the device changes, therefore for new conditions new simulation runs are needed. The quality of simulation results is determined by (i) the validity of the applied model equations and (ii) by the correctness of the applied material parameters and the set of other parameters used by the model equations (e.g. the set of $b$ and $m$ values).

Steps of the validation are the following:

1. Measurement of the small OLEDs and parameter fitting for the creation of the device model equation as it was described in Section 4.

2. SUNRED model building and simulation for the small OLEDs, comparing measurement and simulation results.

3. Measurement of the large OLED.

4. SUNRED model building for the large OLED by using the device model equation of the small OLEDs; simulation of the model and comparing simulation and measurement results.
Fig. 13 shows the SUNRED model of the two investigated OLEDs. A LEP thickness of $60 \mathrm{~nm}$ and ambient temperatures of 10 and $25^{\circ} \mathrm{C}$ were chosen for the presentation of the validation.

Eq. (7a) model equation describes the behavior of the small OLEDs. In case of the large OLED the equation must be modified because the surface area is larger thus, (11a) was applied. The substitution resulted eq. (11b). This equation was used in the simulation of the large OLED.

$$
I_{\text {large }}=I_{\text {small }} \frac{A_{\text {large }}}{A_{\text {small }}}
$$

$$
\begin{aligned}
& I_{l \text { arge }}(U, T)= \\
& \quad\left(2.5 \cdot T^{2}-34.4 \cdot T+593\right) \cdot 10^{-9} \cdot U^{1.4 \cdot 10^{-4} \cdot T^{2}-2.55 \cdot 10^{-2} \cdot T+7.28}
\end{aligned}
$$

Fig. 14 presents the comparison of the measured and simulated curves. The third curve for each temperature is the result of the solution of (7a) for the small OLED and (11b) for the large OLED. In case of the small OLED the three curves cover each other with small error i.e. the model is suitable for further usage.

Though the simulation is based on the model equations, in case of the large OLED, despite the diverging measured and model equation curves, the measured and the simulated curves correlate well, the error is small. The difference of the measured curve and the curve calculated from eq. (11b) is linear except at small currents as presented in Fig. 15. The equations of the linear sections can also be seen in the figure: the large

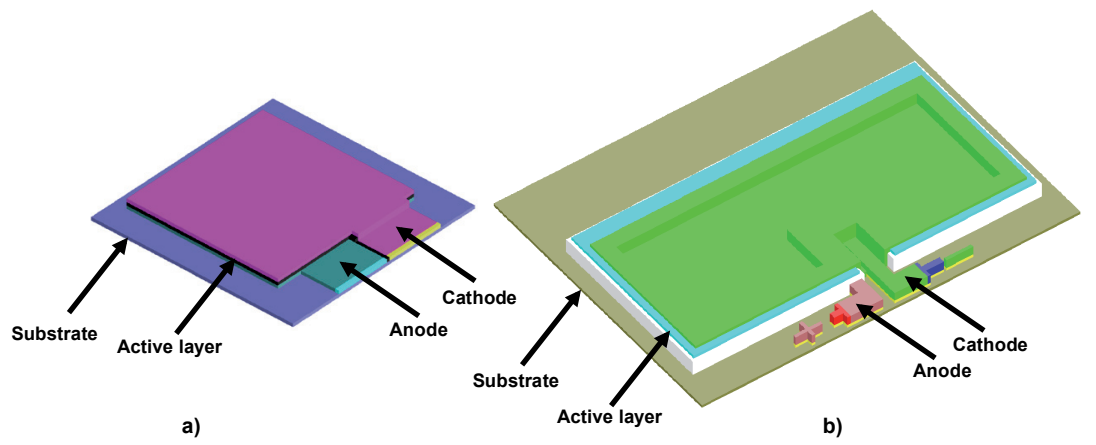

Fig. 13. SUNRED model of the OLED devices (a) Small OLED (b) Large OLED

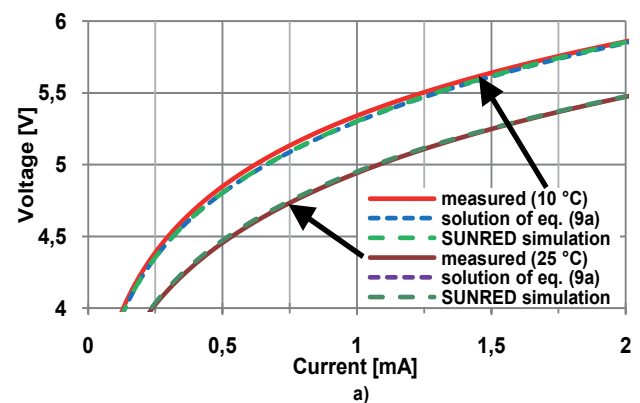

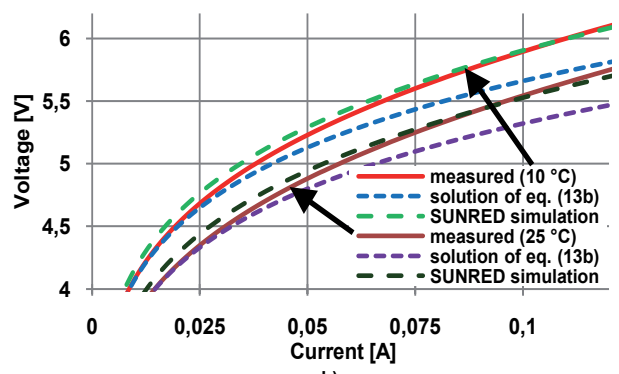

b)

Fig. 14. Comparing measured and simulated results (a) Small OLED (b) Large OLED 


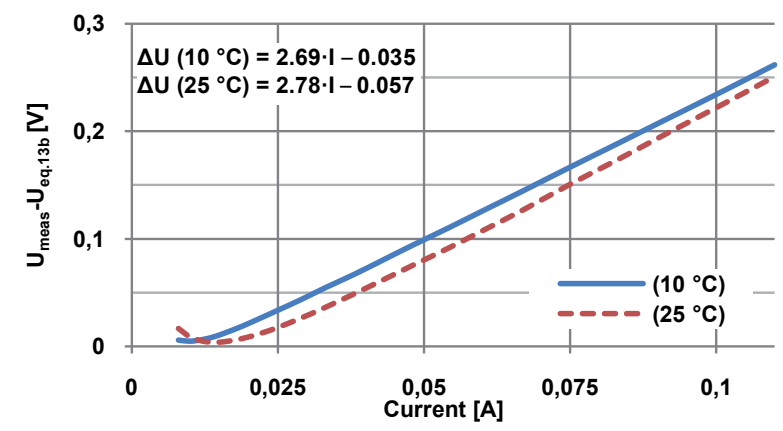

Fig. 15. The difference of the measured curve and the curve calculated from eq. (11b) in case of the large OLED, with the equations of the linear sections

OLED has a different structure than the small one has, that results in a serial resistance of about $2.7 \Omega$. The SUNRED model takes into account the specificities of the large OLED that results in better correlation.

The error between the measured and simulated curves is shown in Fig. 16. The slope of the curve i.e. the serial resistance changes with the temperature, at $10^{\circ} \mathrm{C}$ it is $1.03 \Omega$, at $25^{\circ} \mathrm{C}$ it is $1.49 \Omega$. This is a consequence of the distributed model. Fig. 17 presents the current density distribution in the large OLED at $100 \mathrm{~mA}$ driving current. The distribution is slightly different at 10 and at $25^{\circ} \mathrm{C}$.

The error in the studied voltage domain of the large OLED was in the -2.16 to $+0.56 \%$ range. The maximum error occurred at $19 \mathrm{~mA}, 25^{\circ} \mathrm{C}$ where the measured voltage was $4.16 \mathrm{~V}$ and the simulated voltage was $4.25 \mathrm{~V}$. In practice this error is absolutely acceptable.

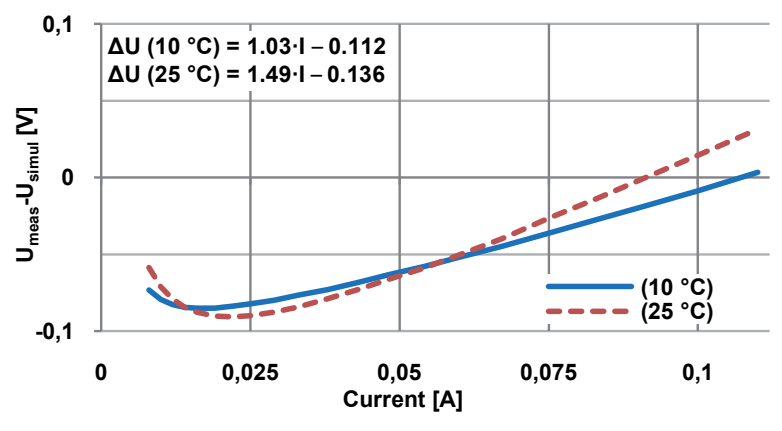

Fig. 16. Relative error of the simulated voltage compared to the measured voltage in case of the large OLED, with the equations of the linear sections

The measured and the simulated temperature distribution were shown in Fig. 3 (b) and (c). The difference between the maximum measured and simulated temperature was under $5 \%$ and the correlation of the measured and simulated distribution is acceptable thus, the thermal model of the large OLED is appropriate for further application.

Fig. 18 compares the results of optical measurements and optical simulations of the large OLEDs. For greater clarity, the values are shown at only one temperature; the overlapping part of the measured and the simulated curves has similarly low deviation at every temperature. Mean-square deviation of the measured and simulated values is between 0.2 and $0.7 \%$ at every temperature. This result suggests that the model and the simulation can be used in OLED design.

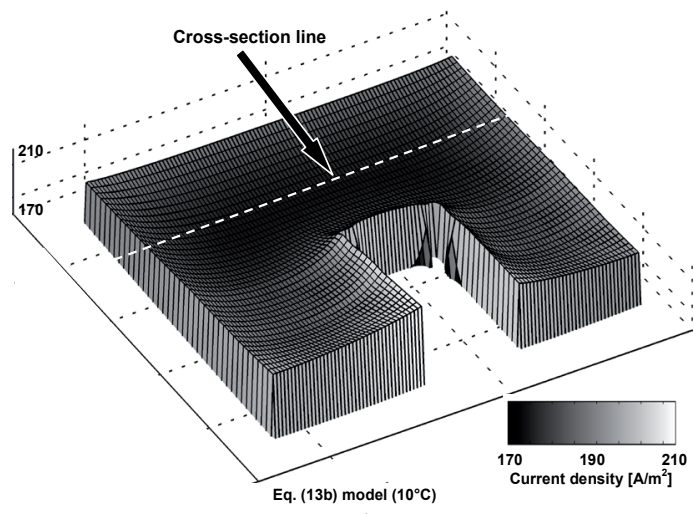

a)

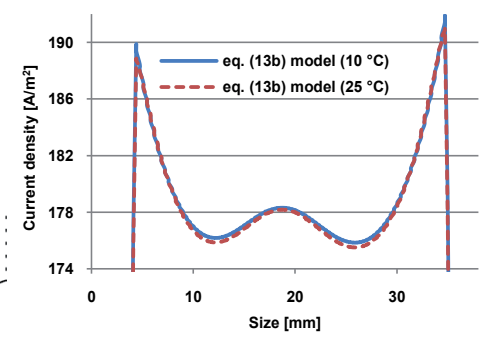

Fig. 17. Current density in the active layer of the large OLED at $100 \mathrm{~mA}$ (a) full surface (b) cross-section curves 


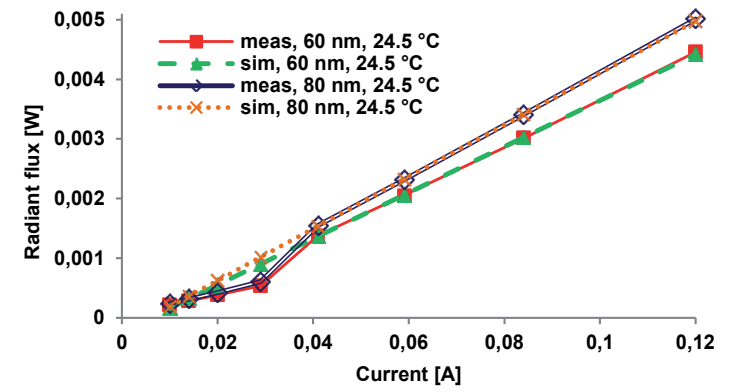

Fig. 18. I-L characteristics: measured vs. simulated results (large OLEDs)

\section{Conclusion}

Electro-thermal and temperature-dependent optical modeling and distributed simulation of large area lighting purpose OLEDs were presented. The extended algorithm of our field solver is able to model large area semiconductor junctions, such as LEP layers of OLEDs properly. The extension can handle any nonlinear function as semiconductor junction equation; the demonstrated electro-thermal OLED model uses power function to describe the junction. The simulator can produce radiance and luminance maps and it can determine the full radiant flux and luminous flux of the light of the simulated devices.

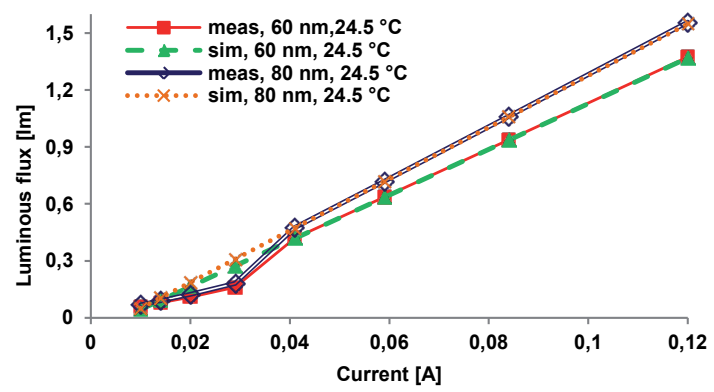

The temperature-dependence of luminous flux is significant; in case of our samples it was $5-22 \%$ after a $40^{\circ} \mathrm{C}$ temperature change, depending on the current.

Writing of a second article is planned in which we will complete the validation of the models by presenting more simulated and measured results including optical measurements. More case studies will also be presented.

We believe that the demonstrated simulator is well usable in the design of large area OLED devices, and in other projects where large area semiconductor devices are examined such as solar cells.

\section{Acknowledgements}

The presented field solver is based on prof. Dr. Vladimir Székely's original SUNRED field solver algorithm [13]. We would like to thank his guidance, recommendations and ideas.

This work has been supported by the ICT-2007.3.2/216641 Fast2Light Project of the Framework 7 program of the EU. This work is connected to the scientific program of the "Development of quality-oriented and harmonized R+D+I strategy and functional model at BME" project. The support of the Hungarian Government through the TÁMOP-4.2.1/B-09/1/KMR-2010-0002 project at the Budapest University of Technology and Economics is also acknowledged.

We wish to express our gratitude for the OLED samples received from H. Lifka (Philips Research Laboratories) and for Mentor Graphics Micred Division for providing TERALED measurement equipment. We also thank G. Hantos (Department of Electron Devices, BME) for the measurements he carried out to support our work.

\section{References}

1 Nakamura S., Zn-doped InGaN growth and InGaN/AlGaN doubleheterostructure blue-light-emitting diodes. Journal of Crystal Growth, 145 (1-4), pp. 911-917, (1994). DOI: $10.1016 / 0022-0248(94) 91163-0$

2 Bando K., Sakano K., Noguchi Y., Shimizu Y., Development of high-bright and pure-white LED lamps. Journal of Light and Visual Environment, 22(1), pp. 2-5, (1998).

3 http://www.cdtltd.co.uk/press/archive_press_release_index/2007/602. asp, March 26, 2007

4 Solid-State Lighting R\&D Manufacturing Roadmap, July 2010, http://www1.eere.energy.gov/buildings/ssl/techroadmaps.html

5 Müllen K., Scherf U., Organic Light Emitting Devices: Synthesis, Properties and Applications. John Wiley \& Sons, (2006).

6 EU FW7 Fast2Light project. http://www.fast2light.org/
7 Krummacher B. C., Nowy S., Frischeisen J., Klein M., Brütting W., Efficiency analysis of organic light-emitting diodes based on optical simulation. Organic Electronics, 10 (3), pp. 478-485, (2009). DOI: $\underline{\text { 10.1016/j.orgel.2009.02.002 }}$

8 Quirino W. G., Teixeira K. C., Legnani C., Calil V. L., Messer B., Vilela Neto O. P., Pacheco M. A. C., Cremona M., Improved multilayer OLED architecture using evolutionary genetic algorithm. Thin Solid Films, 518 (5), pp. 1382-1385 (2009). DOI: $10.1016 /$ j.tsf.2009.09.117

9 Slootsky M., Forrest S. R., Full-wave simulation of enhanced outcoupling of organic light-emitting devices with an embedded lowindex grid. Applied Physics Letters, 94, 163302, (2009). DOI: $\underline{10.1063 / 1.3116644}$ 
10 SimOLED - OLED simulation software http://www.sim4tec. com/?Products:SimOLED _ OLED simulation software

11 Ansys Inc. http://www.ansys.com/

12 Park J., Lee J., Shin D., Park S., Luminance Uniformity of LargeArea OLEDs With an Auxiliary Metal Electrode. Journal of Display Technology, 5(8), pp. 306-311, (2009).

13 Székely V., SUNRED: a new thermal simulator and typical applications. 3rd THERMINIC Workshop, pp. 84-90, (1997).

14 Kollár E., Zólomy I., Poppe A., Electro-thermal modeling of largesurface OLED. in 'Proceedings of the Symposium on Design, Test, Integration and Packaging of MEMS/MOEMS (DTIP'09). Rome, Italy, (2009)' pp. 239-242. (ISBN: 978-2-35500-009-6)

15 Komoda T., Ide N., Kido J., High Efficient OLEDs and Their Application to Lighting. Journal of Light \& Visual Environment, 32(2), pp. 75-78 (2008).

16 Besançon R. M., The Encyclopedia of Physics. Third Edition. Van Nostrand Reinhold Company (1985).

17 Beer G., Watson J. O., Introduction to finite and boundary element methods. John Wiley \& Sons, Chichester (1992).
18 Polyanin A. D., Handbook of Linear Partial Differential Equations for Engineers and Scientists. Chapman \& Hall/CRC Press, Boca Raton (2002).

19 Pohl L., Kohári Zs., Székely V., Fast field solver for the simulation of large-area OLEDs. Microelectronics Journal, 41(9), pp. 566-573, (2010).

DOI: $\underline{10.1016 / \text { j.mejo.2009.12.007 }}$

20 http://www.mentor.com/products/mechanical/products/teraled

21 CIE Technical Report 127:2007, Measurement of LEDs. ISBN: 9783901906589

22 Pohl L., Kollár E., Poppe A., Nonlinear electro-thermal OLED model in SUNRED field simulator. in 'Thermal Investigations of ICs and Systems (THERMINIC), 2010 16th International Workshop on' pp. 149-153 (2010).

23 Demidovich B. P., Maron I. A., Computational Mathematics. Editorial Mir, Moscow, pp. 459-487 (1973). 